\title{
Schwermetalle (Fe, Mn, Cd, Cr, Cu, Pb, Zn) im Pelagial des Bodensees (Obersee und Untersee) und des Greifensees
}

\author{
Von HANS-RUdOLF HEGI \\ Eidgenössische Technische Hochschule Zürich \\ Eidg. Anstalt für Wasserversorgung, Abwasserreinigung und Gewässerschutz (EAWAG) \\ Manuskript eingegangen am 29.Januar 1976
}

\begin{abstract}
Heavy Metals (Fe, $\mathrm{Mn}, \mathrm{Cd}, \mathrm{Cr}, \mathrm{Cu}, \mathrm{Pb}$ and $\mathrm{Zn}$ ) in the Pelagic Zone of the Lake of Constance (Obersee and Untersee) and the Greifensee

Monthly measurements were made over a one-year period to determine the heavy metals $\mathrm{Fe}, \mathrm{Mn}, \mathrm{Cd}$, $\mathrm{Cr}, \mathrm{Cu}, \mathrm{Pb}$ and $\mathrm{Zn}$ in the vertical profiles of Lake Constance (Obersee and Untersee) and Greifensee, by means of atomic absorption spectrometry. Fe and Mn exhibited the usual cyclical fluctuations depending on redox conditions in the hypolimnion. For $\mathrm{Cd}, \mathrm{Cr}, \mathrm{Cu}, \mathrm{Pb}$ and $\mathrm{Zn}$ no concentration patterns were noted that would show their dependency on seasonal and vertical stratifications.
\end{abstract}

\section{Einleitung}

\subsection{Allgemeines}

Die Kontamination unseres Lebensraumes mit Schwermetallen hat einen Komplex von ökologischen Fragen hervorgebracht, welche eine weit gestreute analytische Aktivität im Gefolge haben. Während die Bereiche der Flüsse, Ästuarien, Meeresküsten und auch offener Ozeane bereits recht häufig auf Schwermetalle untersucht worden sind, wie aus zahlreichen Publikationen hervorgeht, sind Binnenseen in dieser Hinsicht bis jetzt noch verhältnismässig wenig untersucht worden (vgl. Abschnitt 1.3). In der vorliegenden Mitteilung werden Analysenwerte bekanntgegeben, welche im Rahmen von monatlichen Routineuntersuchungen der «Internationalen Gewässerschutzkommission für den Bodensee» und interner Programme durch die EAWAG ausgeführt worden sind. In das Analysenprogramm wurde neben der Bestimmung von Eisen und Mangan die Bestimmung derjenigen Schwermetalle aufgenommen, die weitherum durch zivilisatorische Aktivitäten in die Gewässer gelangen und deren analytische Erfassung in den allenfalls zu erwartenden Konzentrationen mit einer einfachen, generell anwendbaren Messmethodik als aussichtsreich erschien. Die Bestimmung erfolgte in sämtlichen Proben, die anlässlich der konventionellen chemischen Seenuntersuchung ohnehin gefasst wurden, d.h. 
im Tiefenprofil in monatlichen Intervallen. $\mathrm{Ob}$ sich aus den Analysendaten schliesslich Zusammenhänge irgendwelcher Art, hinsichtlich der Schwermetalle untereinander oder gegenüber anderen Parametern - auch räumlich oder zeitlich herausarbeiten lassen würden, konnte zu Beginn der Untersuchung noch keineswegs abgeschätzt werden; mit Ausnahme von Eisen und Mangan wurde weitgehend Neuland betreten.

\subsection{Untersuchte Gewässer, Probenahmeorte, Zeitplan}

Das Probenahmeprogramm umfasste Seen vom oligo-mesotrophen (BodenseeObersee), vom eutrophen (Bodensee-Untersee) und vom hoch eutrophen Typus (Greifensee). Der Bodensee-Obersee wird im folgenden als «Obersee» bezeichnet, der Bodensee-Untersee als «Untersee». Die Orte der Probenahme (Seemitte, bzw. tiefste Stelle des betreffenden Seebeckens) sowie die wichtigsten morphometrischen Daten, ferner die Dauer der Untersuchungsperioden sind in Tabelle 1 zusammengestellt.

Die Proben wurden von folgenden Instituten erhoben:

Obersee: Staatliches Institut für Seenforschung und Seenbewirtschaftung, Abt. Max-Auerbach-Institut Konstanz, zusammen mit EAWAG, Abt. Limnologie

Untersee: Landesanstalt für Umweltschutz Baden-Württemberg, Institut für Seenforschung und Fischereiwesen, Insel Reichenau

Greifensee: EAWAG, Abt. Limnologie

\subsection{Bisherige Kenntnisse über Schwermetalle im Seenpelagial}

Neuere Daten über die jahreszyklische Vertikalverteilung von Fe und Mn in Seewasser sind von folgenden Autoren bekanntgegeben worden: BürGI [5] ermittelte einen Jahreszyklus des Eisens im Greifensee und fand für das Gesamteisen im Tiefenwasser v. a. während der Stagnationsphasen eine Anreicherung. BLoESCH [3] hat im Rotsee ähnliche Verhältnisse festgestellt, währenddem er im Vierwaldstättersee ausserdem ein epilimnisches Maximum fand; er bestimmte auch die Mn- und FeGehalte in den Sedimenten und beobachtete vor allem beim $\mathrm{Mn}$, weniger sicher auch beim $\mathrm{Fe}$ eine Abhängigkeit dieser Gehalte von der jahreszyklischen Vertikalverteilung, die zum mindesten beim Mn auf dessen Rücklösung aus dem sauerstofffreien Sediment schliessen lässt. DeLfINo und LeE [7] untersuchten den Jahreszyklus des Lake Mendota, Wisconsin, und fanden ein Ansteigen des Mn-Gehaltes parallel zur Entwicklung der thermischen Schichtung im Frühsommer, wobei der Mn-Gehalt vom Gehalt an gelöstem Sauerstoff eindeutig abhängig war: bei Sauerstoffgehalten von über $2 \mathrm{mg} \mathrm{O} / 1$ lagen die Mn-Gehalte unter $0,05 \mathrm{mg} / \mathrm{l}$, bei Sauerstoffgehalten von unter $2 \mathrm{mg} \mathrm{O} / 1$ lagen sie zwischen 0,2 und $0,8 \mathrm{mg} \mathrm{Mn} / 1$. GrotH [12] fand in holsteinischen Seen ebenfalls vor allem beim Mn eine deutliche, beim $\mathrm{Fe}$ eine weniger deutliche Abhängigkeit vom Sauerstoffgehalt. AмBÜHL [1] gab den Verlauf des Sauerstoffgehaltes (Zeit-Tiefen-Diagramm) im Bodensee-Untersee während mehrerer Jahre bekannt.

Uber Gehalte an weiteren Schwermetallen in Seen erfolgte eine ausführlichere Bekanntgabe durch folgende Autoren: BRADFORD et al. [4] ermittelten in 170 Seen 
Kaliforniens $\mathrm{Cu}, \mathrm{Pb}, \mathrm{Ni}, \mathrm{Zn}$. Sie gaben als Erfassungsgrenze der Bestimmung für alle genannten Metalle $0,3 \mu \mathrm{g} / 1$ an, als Bereich der ermittelten Konzentrationen für $\mathrm{Cu} 0,4 \ldots 5, \mathrm{~Pb} 0,3 \ldots 4, \mathrm{Ni} 0,3 \ldots 0,8, \mathrm{Zn} 0,3 \ldots 100 \mu \mathrm{g} / \mathrm{l}$, als Medianwerte für $\mathrm{Cu} \mathrm{1,2}$, $\mathrm{Pb} 0,5$, Ni 0,3, Zn 1,5 $\mu \mathrm{g} / 1$. Groth [12] untersuchte die jahreszyklische Vertikalverteilung von $\mathrm{Co}, \mathrm{Cu}, \mathrm{Mo}, \mathrm{Zn}$ im Schöhsee. In bezug auf die Gesamtgehalte an den genannten Metallen hat er - ausser teilweise höheren Gehalten im Hypolimnion während der sommerlichen Stagnationsphase - keine oder nur undeutlich erkennbare Zusammenhänge mit der periodischen Stratifikation gefunden. Die durchschnittlichen Konzentrationen innerhalb der Untersuchungsperiode betrugen ungefähr: $\mathrm{Co}$ 0,05, Cu 1, Mo 0,3, Zn $2 \mu \mathrm{g} / \mathrm{l}$. KIMBALL [15] untersuchte die jahreszyklische Verteilung des $\mathrm{Cu}$ im Knights Pond (Tiefe maximal $4 \mathrm{~m}$ ) und fand Werte zwischen $<10$ und $105 \mu \mathrm{g} \mathrm{Cu} / 1$; sie lagen im Winter höher als im Sommer. WELz und WiEDEKING [21] nahmen für $\mathrm{Cr}, \mathrm{Cu}$ und andere Metalle im Bodensee-Obersee und im Mindelsee je ein einziges Vertikalprofil auf und fanden in membranfiltrierten Proben aus dem Obersee $\mathrm{Cr}-\mathrm{Gehalte}$ zwischen 1,5 und $6 \mu \mathrm{g} / \mathrm{l}$ und $\mathrm{Cu}-\mathrm{Gehalte}$ zwischen 3 und $6 \mu \mathrm{g} / \mathrm{l} ; \mathrm{Pb}$ lag in allen Tiefen unter der Nachweisgrenze von $0,2 \mu \mathrm{g} / \mathrm{l}$.

\section{Methoden}

\subsection{Probenahme und -konservierung}

Die Seewasserproben wurden mit den Vertikal-Wasserschöpfern der beteiligten Institute erhoben; sämtliche Teile, welche mit Wasser in Kontakt kommen, sind aus Kunststoffen gefertigt oder mit solchen beschichtet (PVC, Plexiglas, PTFE). Die Drahtseile der verwendeten Winden bestehen aus Chromstahl.

Aus den Wasserschöpfern wurde jeweils ein Probenanteil von ca. $100 \mathrm{ml}$ direkt, d.h. ohne jegliche Vorbehandlung, in die Probenflaschen abgefüllt. Als Probenflaschen wurden 100-ml-Sovirelflaschen (Pyrexglas) mit Schraubverschluss und Tefloneinlage verwendet; die Flaschen gelangten ausschliesslich für Schwermetallbestimmungen in Seewasserproben zum Einsatz und wurden vor den Probenahmen jeweils mit verdünnter Salpetersäure gereinigt. Nach dieser Reinigung, aber vor der Probenahme, wurden sie mit $2 \mathrm{ml} 5 n$-Salpetersäure (aus Salpetersäure suprapur «Merck») versetzt; in der Probe lag die Säure somit in der Konzentration 0,1normal vor. Die Proben wurden bis zu den Messungen bei Zimmertemperatur im Labor stehengelassen; die Messungen erfolgten nach Möglichkeit innert weniger Tage nach der Probenahme.

Die Probenkonservierung mit Säure ist notwendig, da ohne Konservierungsmittelzugabe beim Stehen der Proben Verluste an Metallen auftreten können $[17,19]$.

\subsection{Messtechnik}

Die Messungen wurden in der Regel mit flammenloser Atomabsorption (Graphitrohr), in Einzelfällen - bei höheren Konzentrationen an Eisen und Mangan mit Flammenatomabsorption durchgeführt. Geräte: Atomabsorber PerkinElmer 305 mit Graphitrohrküvette HGA-70 für Graphitrohrmessungen, Atomabsorber Perkin-Elmer 306 für Flammenmessungen. Die Standardlösungen wurden in $0,1 n$-Salpetersäure angesetzt. 


\subsection{Bemerkungen zur Methodik}

Es stellt sich die Frage, ob mit der angewandten Probenkonservierungs- und Messmethodik die einzelnen Metalle vollständig erfasst werden. Dazu ist zu sagen, dass die ursprünglich bereits gelösten sowie die mit der zugesetzten Salpetersäure in Lösung gehenden Metallanteile mit Sicherheit quantitativ, die in der Säure noch suspendierten Metallanteile möglicherweise nur zu einem unbestimmten Anteil zur Atomisierung und somit zur Messung gelangen. Wir haben indessen festgestellt, dass sich sogar die in getrocknetem Schwebstoffmaterial bzw. in Sedimenten enthaltenen Schwermetalle in $0,1 n$-Salpetersäure weitgehend lösen; bei im Wasser suspendiertem Schwebstoffmaterial dürfte dies erst recht der Fall sein.

In Oberflächenwässern werden Schwermetalle oftmals nur nach vorgängiger Filtration (meist Membranfilter $0,45 \mu \mathrm{m}$ ) oder sowohl in unfiltrierten als auch in filtrierten Probenanteilen bestimmt [6, 14, 16]. Wir haben von Metallbestimmungen in filtrierten Proben abgesehen, nachdem es sich schon bald gezeigt hatte, dass die Gehalte an $\mathrm{Cd}, \mathrm{Cu}$ und $\mathrm{Pb}$ bereits in unfiltrierten Proben in der Mehrzahl der Fälle unter der Erfassungsgrenze lagen und da uns von Untersuchungen an Fliessgewässern her bekannt war, dass bei niedrigen Metallgehalten die Filtration zu Schwierigkeiten, z. B. zu Kontaminationen durch das Filtriergerät führen kann.

Selbst bei der Messung in unfiltrierten Proben hält es schwer, sämtliche Möglichkeiten der Kontamination, vor allem mit Zink, sicher auszuschalten.

Zwar wurden die Probenahmegeräte, die Probenflaschen, die zur Konservierung verwendete Salpetersäure und die Pipettenspitzen auf mögliche Kontaminationsgefahren untersucht. Dennoch lässt sich die Möglichkeit nicht ausschliessen, dass sporadisch und unregelmässig aufgetretene, verhältnismässig hohe Metallgehalte durch Kontamination zustandegekommen sind. Dies mit Sicherheit feststellen bzw. ausschliessen zu können hätte die häufige oder regelmässige Entnahme mehrerer Parallelproben erfordert, was im Rahmen des Routineprogrammes aber nicht möglich war.

\section{Untersuchungsresultate}

Die Resultate der Metallbestimmungen sind in den Tabellen 3 bis 7 zusammengestellt. $\mathrm{Fe}, \mathrm{Mn}, \mathrm{Cu}$ und $\mathrm{Zn}$ sind als Einzelwerte angegeben, $\mathrm{Cd}, \mathrm{Cr}$ und $\mathrm{Pb}$ lediglich in Form der Häufigkeitsverteilung aller Werte, da bei diesen drei Metallen ein erheblicher Anteil der Messwerte unter der Erfassungsgrenze der angewandten Bestimmungsmethode lag und das Auftreten der wenigen höherliegenden Werte keinen örtlichen oder zeitlichen Zusammenhang erkennen lässt.

\section{Diskussion}

\subsection{Eisen und Mangan}

Beim Fe und vor allem beim Mn zeigte sich eine jahreszyklische Abhängigkeit der Gehalte von den Redoxverhältnissen in den Tiefenschichten, wie sie von bereits erwähnten und von früheren Untersuchungen her bekannt ist $[3,5,7,12]$. Beim Greifensee und - weniger ausgeprägt - beim Untersee waren im sauerstofffreien 
bzw. -armen Hypolimnion erhöhte Mn-Werte, teilweise auch erhöhte Fe-Werte zu erkennen, während beim Oberseeprofil der minimale Sauerstoffgehalt im Verlaufe der Untersuchung $6,6 \mathrm{mg} \mathrm{O} / 1$ (7.Januar 1975) betrug und mithin auch kein Ansteigen der $\mathrm{Mn}$ - und $\mathrm{Fe}$-Gehalte erfolgte.

\subsection{Cadmium, Chrom, Kupfer, Blei, Zink}

Ein erheblicher Teil der Messdaten von $\mathrm{Cd}, \mathrm{Cr}, \mathrm{Cu}$ und $\mathrm{Pb}$ lag unter den jeweiligen Erfassungsgrenzen; beim Zn lagen alle Werte darüber. Bei keinem der genannten Metalle ist indessen ein Konzentrationsverlauf festzustellen, der eine Abhängigkeit von Jahreszeit und vertikaler Schichtung oder sonst irgendeine Stetigkeit erkennen liesse. Im Gegenteil ist das Auftreten von Gehalten, die eindeutig oder erheblich über den Erfassungsgrenzen liegen, durch Unregelmässigkeit gekennzeichnet und zwar im Verlaufe der ganzen Untersuchung. Die Gehalte an $\mathrm{Cu}$ und $\mathrm{Zn}$ liegen beim Untersee erheblich höher als beim Obersee und beim Greifensee. Die Unterschiede zwischen den Gehalten in Obersee und Untersee dürften sich durch die erhöhte Belastung des Untersees mit den genannten Metallen als Folge der Anwendung von Pflanzenschutzmitteln bzw. als Folge von Abwassereinleitungen erklären lassen. Wir haben mit EDV eine Korrelationsanalyse zwischen den Metallen $\mathrm{Cd}, \mathrm{Cr}, \mathrm{Cu}, \mathrm{Pb}, \mathrm{Zn}$ und weiteren Komponenten (Temperatur, Leitfähigkeit, Sauerstoff, pH, Kohlensäure, Carbonathärte, Gesamthärte, Kalzium, Kieselsäure, Orthophosphat, Gesamtphosphor, Ammonium, Nitrit, Nitrat, Kjeldahlstickstoff, Chlorid, Sulfat, organischer Kohlenstoff, assimilierter C, Sulfid, Sichttiefe) ausgeführt. Sie ergab, dass zwischen den genannten Schwermetallen unter sich sowie zwischen den Schwermetallen und den übrigen Komponenten die Quadrate der linearen Korrelationskoeffizienten in jedem Falle kleiner als 0,3 sind, mit Ausnahme von $\mathrm{Cr} / \mathrm{Pb}$ und $\mathrm{Cr} / \mathrm{Nitrat}$.

Wie bereits erwähnt, ist eine jahreszyklische Regelmässigkeit in der Vertikalverteilung der Schwermetalle nicht zu erkennen. Naheliegend ist die Frage, welche Faktoren zu einer solchen Rhythmik hätten führen können. Am ehesten hätten dies einerseits die Sedimentation von Schwermetallen und anderseits deren Rücklösung aus sedimentiertem Material sein können $[2,9,11,18,20]$. Seesedimente enthalten nämlich erhebliche Anteile an einigen Schwermetallen. Wir fanden in einer Sedimentprobe aus dem Greifensee (Abschnitt eines Sedimentbohrkerns, aus $7 \mathrm{~cm}$ Tiefe unter der Sedimentoberfläche) in $\mathrm{mg}$ Metall/kg Trockensubstanz: Fe 12100 , $\mathrm{Mn} \mathrm{450;} \mathrm{Cd} \mathrm{5;} \mathrm{Cu} \mathrm{30;} \mathrm{Cr} \mathrm{20;} \mathrm{Pb} \mathrm{70;} \mathrm{Zn} \mathrm{100.} \mathrm{Auch} \mathrm{von} \mathrm{Sedimentuntersuchungen}$ aus weiteren Seen sind Schwermetallgehalte in diesen Grössenordnungen bekannt $[8,11,12,13]$. Da die Sedimentbildung, z. B. die Ausfällung von Kalziumkarbonat, die biogene Sedimentation von Phosphaten usw. von der jeweiligen Produktivität der Seen in den oberen Wasserschichten und somit auch von der Jahreszeit abhängt, könnte auch die mit der Sedimentation einhergehende Elimination der Schwermetalle aus dem Wasser jahreszeitlichen Schwankungen unterliegen. Umgekehrt ist auch eine Remobilisierung von Schwermetallen aus den Sedimenten denkbar. Es ist bekannt, dass $\mathrm{Mn}$ und $\mathrm{Fe}$ unter geeigneten Bedingungen aus Sedimenten remobilisiert werden $[3,7,12,20]$. Da zudem unter anderem höher oxidierte Eisenund Manganoxidhydrate als Spurenfänger für weitere Metalle wirken können 
$[11,13,20]$, ist es durchaus möglich, dass bei allfälliger Remobilisierung von Mn bzw. Fe die ursprünglich mitgefällten Schwermetalle ebenfalls wieder in Lösung gehen. Es hätten somit beim Auftreten erhöhter Konzentrationen an Fe und Mn im Seewasser, nämlich im Hypolimnion vom Greifensee und vom Untersee während der sommerlichen Stagnationsphase, auch die Konzentrationen an weiteren Schwermetallen erhöht sein können.

Die Ermittlung der Schwermetallgehalte im Seewasser wäre auch im Rahmen der Aufstellung einer auf die Seen bezogenen Bilanz von Interesse. Das dazu erforderliche Datenmaterial fehlt aber gegenwärtig noch. Neben den Frachtwerten für Import aus Zuflüssen und Niederschlägen sowie Export aus dem See wären dazu Kenntnisse über den Sedimentationsverlust erforderlich. Gegenwärtig laufende Forschungsprojekte sollen diese Lücke in absehbarer Zeit schliessen.

\section{Zusammenfassung}

Von Anfang 1974 bis Frühjahr 1975 wurden die Schwermetalle Fe, Mn, Cd, Cr, $\mathrm{Cu}, \mathrm{Pb}, \mathrm{Zn}$ monatlich in Vertikalprofilen vom Bodensee-Obersee, Bodensee-Untersee und Greifensee mittels Atomabsorptionsspektrometrie (meist flammenlos) bestimmt.

$\mathrm{Fe}$ und vor allem Mn zeigten beim Bodensee-Untersee und beim Greifensee (beide eutroph, mit $\mathrm{O}_{2}$-Schwund) die bekannte jahreszyklische Abhängigkeit von den Redoxverhältnissen im Hypolimnion. Beim oligo-mesotrophen Bodensee-Obersee war ein entsprechendes Ansteigen der Fe- und Mn-Gehalte nicht zu erkennen.

Bei den Gehalten an $\mathrm{Cd}, \mathrm{Cr}, \mathrm{Cu}, \mathrm{Pb}, \mathrm{Zn}$ war kein Konzentrationsverlauf festzustellen, der eine Abhängigkeit von Jahreszeit und vertikaler Schichtung oder sonst irgendeine Stetigkeit erkennen liesse. Die Gehalte an $\mathrm{Cd}, \mathrm{Cr}, \mathrm{Cu}, \mathrm{Pb}$ lagen zu einem erheblichen Anteil der gemessenen Werte unter den jeweiligen Erfassungsgrenzen ( $\mathrm{Cd} \mathrm{0,1,} \mathrm{Cr} 0,2, \mathrm{Cu} 1, \mathrm{~Pb} 1, \mathrm{Zn}$ ca. $1 \mu \mathrm{g} / \mathrm{l}$ unter den eingehaltenen Messbedingungen).

Die Gehalte an $\mathrm{Cu}$ und $\mathrm{Zn}$ lagen beim Bodensee-Untersee erheblich höher als beim Bodensee-Obersee und beim Greifensee; die Gehalte an $\mathrm{Cd}, \mathrm{Cr}, \mathrm{Pb}$ in den drei Seen waren indessen nicht deutlich voneinander verschieden.

Tabelle 1. Morphometrie der Seen; Probenahmestellen und Zeitraum der Untersuchungen. Table 1. Morphometry of the lakes; sampling sites and duration of investigation.

\begin{tabular}{|c|c|c|c|c|c|c|}
\hline See & \multicolumn{2}{|l|}{$\begin{array}{l}\text { Seetiefe } \\
\text { mittlere m }\end{array}$} & $\begin{array}{l}\text { Seetiefe } \\
\text { maximale m }\end{array}$ & \multicolumn{2}{|c|}{$\begin{array}{l}\text { Seeflăche } \\
\text { km }^{2}\end{array}$} & $\begin{array}{l}\text { Volumen } \\
\mathbf{k m}^{3}\end{array}$ \\
\hline Obersee & 100 & & 252 & 476 & & 47,6 \\
\hline Untersee & 13 & & 47 & 64 & & 0,83 \\
\hline Greifensee & 19 & & 34 & 8 & & 0,16 \\
\hline See & $\begin{array}{l}\text { Probenahmestelle } \\
\text { Lage }\end{array}$ & Tiefe $\mathrm{m}$ & $\begin{array}{l}\text { Koordinaten } \\
\text { (Landeskarte der }\end{array}$ & Schweiz) & $\begin{array}{l}\text { Zeitr } \\
\text { Prob }\end{array}$ & \\
\hline Obersee & Hagnau-Münsterlingen & 206 & $738000 / 279600$ & & Janu & April 1975 \\
\hline Untersee & Berlingen (Rheinsee) & 45 & $717300 / 281800$ & & Janu & März 1975 \\
\hline Greifensee & Maur-Greifensee & 31 & $693400 / 245400$ & & Janu & ember 1974 \\
\hline
\end{tabular}


Tabelle 2. Messbedingungen bei Anwendung der Graphitrohrküvette.

Table 2. Measuring conditions using a graphite furnace.

\begin{tabular}{lllllllll}
\hline Element & $\begin{array}{l}\text { Wellen- } \\
\text { länge } \\
\mathrm{nm}\end{array}$ & $\begin{array}{l}\text { Einspritz- } \\
\text { volumen } \\
\mu l\end{array}$ & $\begin{array}{l}\text { Thermische } \\
\text { Vorbehand- Atomi- } \\
\text { lung }{ }^{\circ} \mathrm{C}\end{array}$ & $\begin{array}{l}\text { sierungs- } \\
\text { Temp. }\end{array}{ }^{\circ} \mathrm{C}$ & $\begin{array}{l}\text { Skalen- } \\
\text { dehnung }\end{array}$ & Gasstop & $\begin{array}{l}\mathrm{D}_{2-} \\
\text { kompen- } \\
\text { sator }\end{array}$ & $\begin{array}{l}\text { Erfassungs- } \\
\text { grenze } \\
\mu \mathrm{g} / \mathrm{l}\end{array}$ \\
\hline $\mathrm{Cd}$ & 228,8 & 50 & 330 & 1800 & $1 \times$ & + & + & 0,1 \\
$\mathrm{Cr}$ & 357,9 & $2 \times 50$ & 1100 & 2600 & $5 \times$ & - & - & 0,2 \\
$\mathrm{Cu}$ & 324,7 & 50 & 490 & 2400 & $5 \times$ & - & - & 1 \\
$\mathrm{Fe}$ & 248,3 & $20 / 50$ & 750 & 2400 & $2 \times$ & - & - & 1 \\
$\mathrm{Mn}$ & 279,5 & $20 / 50$ & 750 & 2400 & $1 \times$ & - & - & 0,5 \\
$\mathrm{~Pb}$ & 283,3 & 50 & 330 & 2200 & $1 \times$ & + & + & 1 \\
$\mathrm{Zn}$ & 213,9 & 20 & 330 & 1800 & $1 \times$ & - & - & 1 \\
\hline
\end{tabular}

Zeiten: Trocknung $40 \mathrm{sec}$, thermische Vorbehandlung $30 \mathrm{sec}$, Atomisierung $20 \mathrm{sec}$. Temperatur in der Trocknungsphase: $100^{\circ} \mathrm{C}$. Einspritzungen mit Eppendorf-Mikroliterpipetten und entsprechenden Pipettenspitzen. Lieferant: Dr. Vaudaux \& Cie., Postfach, CH-4027 Basel.

Tabelle 3.1. Eisen (Fe), in $\mu \mathrm{g} / 1$. Bodensee-Obersee, Stelle Hagnau-Münsterlingen.

Table 3.1. Iron (Fe), expressed in $\mu g / 1$. Lake Constance-Obersee, site Hagnau-Münsterlingen.

\begin{tabular}{|c|c|c|c|c|c|c|c|c|c|c|c|c|c|c|c|}
\hline & 1974 & & & & & & & & & & & & 975 & & \\
\hline $\begin{array}{l}\text { Monat } \\
\text { Tag }\end{array}$ & $\begin{array}{r}1 . \\
22 .\end{array}$ & $\begin{array}{r}2 . \\
19 .\end{array}$ & $\begin{array}{r}3 . \\
19 .\end{array}$ & $\begin{array}{r}4 . \\
23 .\end{array}$ & $\begin{array}{r}5 . \\
14 .\end{array}$ & $\begin{array}{r}6 . \\
11 .\end{array}$ & $\begin{array}{l}7 . \\
9 .\end{array}$ & $\begin{array}{r}8 . \\
13 .\end{array}$ & $\begin{array}{r}9 . \\
10 .\end{array}$ & $\begin{array}{r}10 . \\
8 .\end{array}$ & $\begin{array}{r}11 . \\
5 .\end{array}$ & $\begin{array}{r}12 . \\
3 .\end{array}$ & 1. & $\begin{array}{l}2 . \\
4 .\end{array}$ & $\begin{array}{l}3 . \\
4 .\end{array}$ \\
\hline \multicolumn{16}{|l|}{ Tiefe $\mathrm{m}$} \\
\hline 0 & 5 & 5 & 3 & 4 & 7 & 11 & 6 & 10 & 4 & 8 & 9 & 4 & 7 & 9 & 6 \\
\hline 5 & 5 & 5 & 3 & 5 & 8 & 6 & 6 & 8 & 9 & 15 & 13 & 4 & 5 & 9 & 8 \\
\hline 10 & 7 & 3 & 3 & 5 & 17 & 6 & 8 & 10 & 9 & 8 & 14 & 4 & 7 & 11 & 7 \\
\hline 20 & 7 & 5 & 3 & 5 & 12 & 6 & 12 & 14 & 4 & 5 & 12 & 3 & 7 & 8 & 7 \\
\hline 30 & 5 & 5 & 4 & 6 & 7 & 4 & 10 & 11 & 5 & 10 & 12 & 5 & 20 & 6 & 11 \\
\hline 50 & 5 & 3 & 3 & 7 & 4 & 10 & 5 & 8 & 4 & 4 & 8 & 3 & 10 & 7 & 9 \\
\hline 100 & 5 & 8 & 5 & 6 & 4 & 4 & 3 & 13 & 4 & 5 & 4 & 5 & 8 & 10 & 7 \\
\hline 150 & 6 & 4 & 10 & 24 & 6 & 4 & 3 & 5 & 5 & 3 & 4 & 4 & 4 & 7 & 10 \\
\hline 180 & 7 & 8 & 6 & 5 & 8 & 9 & 5 & 10 & 5 & 5 & 3 & 2 & 5 & 16 & 10 \\
\hline 200 & 21 & 3 & 10 & 10 & 5 & 4 & 5 & 10 & 6 & 4 & 5 & 5 & 7 & 10 & 8 \\
\hline
\end{tabular}

Tabelle 3.2. Eisen ( $\mathrm{Fe}$ ), in $\mu \mathrm{g} / \mathrm{l}$. Bodensee-Untersee, Stelle Berlingen.

Table 3.2. Iron ( $\mathrm{Fe}$ ), expressed in $\mu \mathrm{g} / \mathrm{l}$. Lake Constance-Untersee, site Berlingen.

1974

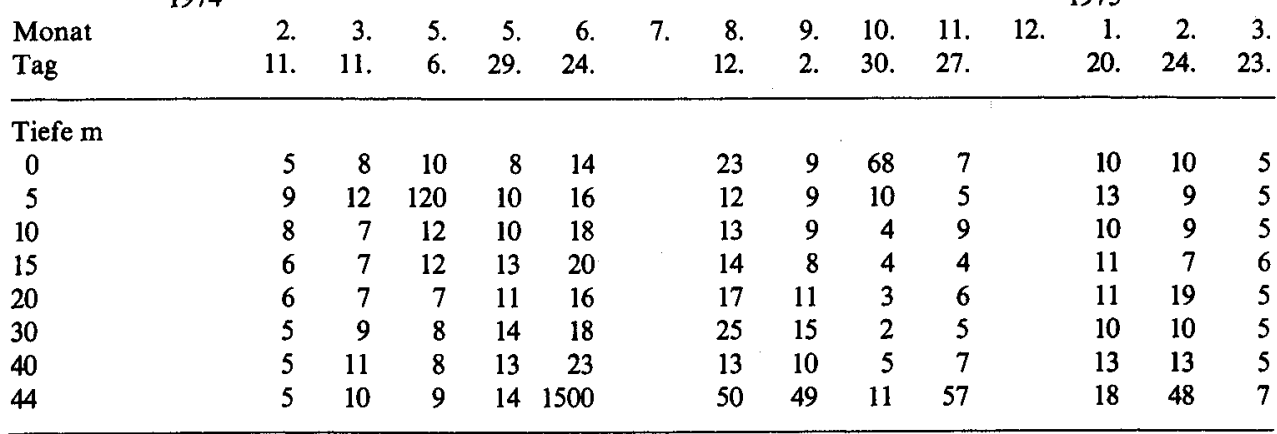


Tabelle 3.3. Eisen ( $\mathrm{Fe})$, in $\mu \mathrm{g} / \mathrm{l}$. Greifensee, Stelle Maur-Greifensee.

Table 3.3. Iron ( $\mathrm{Fe}$ ), expressed in $\mu \mathrm{g} / \mathrm{l}$. Greifensee, site Maur-Greifensee.

\begin{tabular}{|c|c|c|c|c|c|c|c|c|c|c|c|c|c|}
\hline $\begin{array}{l}\text { Monat } \\
\text { Tag }\end{array}$ & $\begin{array}{r}1974 \\
1 . \\
7 .\end{array}$ & $\begin{array}{l}2 . \\
4 .\end{array}$ & $\begin{array}{l}3 . \\
4 .\end{array}$ & $\begin{array}{l}4 . \\
2 .\end{array}$ & $\begin{array}{r}4 . \\
29 .\end{array}$ & $\begin{array}{r}5 . \\
27 .\end{array}$ & $\begin{array}{r}6 . \\
24 .\end{array}$ & $\begin{array}{r}7 . \\
22 .\end{array}$ & $\begin{array}{r}8 . \\
19 .\end{array}$ & $\begin{array}{r}9 . \\
16 .\end{array}$ & $\begin{array}{l}10 . \\
15 .\end{array}$ & $\begin{array}{l}11 . \\
11 .\end{array}$ & $\begin{array}{r}12 . \\
9 .\end{array}$ \\
\hline \multicolumn{14}{|l|}{ Tiefe $m$} \\
\hline 0 & 10 & 21 & 19 & 4 & 11 & 11 & 11 & 18 & 13 & 4 & 13 & 11 & 14 \\
\hline 1 & 17 & 18 & 18 & 10 & 20 & 9 & 12 & 17 & 8 & 6 & 13 & 12 & 16 \\
\hline 2,5 & 17 & 16 & 20 & 4 & 20 & 8 & 15 & 18 & 10 & 9 & 18 & 11 & 17 \\
\hline 5 & 25 & 23 & 18 & 10 & 10 & 7 & 15 & 24 & 6 & 9 & 16 & 11 & 18 \\
\hline 10 & 10 & 25 & 17 & 7 & 6 & 8 & 5 & 24 & 18 & 9 & 13 & 17 & 14 \\
\hline 20 & 21 & 20 & 18 & 9 & 45 & 7 & 12 & 24 & 31 & 19 & 25 & 24 & 16 \\
\hline 30 & 25 & 48 & 19 & 10 & 21 & 14 & 26 & 33 & 78 & 44 & 50 & 40 & 17 \\
\hline
\end{tabular}

Tabelle 4.1. Mangan (Mn), in $\mu \mathrm{g} / 1$. Bodensee-Obersee, Stelle Hagnau-Münsterlingen.

Table 4.1. Manganese (Mn), expressed in $\mu \mathrm{g} /$ l. Lake Constance-Obersee, site Hagnau-Münsterlingen.

\begin{tabular}{|c|c|c|c|c|c|c|c|c|c|c|c|c|c|c|c|}
\hline & 1974 & & & & & & & & & & & & 1975 & & \\
\hline $\begin{array}{l}\text { Monat } \\
\text { Tag }\end{array}$ & $\begin{array}{r}1 . \\
22 .\end{array}$ & $\begin{array}{r}2 . \\
19 .\end{array}$ & $\begin{array}{r}3 . \\
19 .\end{array}$ & $\begin{array}{r}4 . \\
23 .\end{array}$ & $\begin{array}{r}5 . \\
14 .\end{array}$ & $\begin{array}{r}6 . \\
11 .\end{array}$ & $\begin{array}{l}7 . \\
9 .\end{array}$ & $\begin{array}{r}8 . \\
13 .\end{array}$ & $\begin{array}{r}9 . \\
10 .\end{array}$ & $\begin{array}{r}10 . \\
8 .\end{array}$ & $\begin{array}{r}11 . \\
5 .\end{array}$ & $\begin{array}{r}12 . \\
3 .\end{array}$ & $\begin{array}{l}1 . \\
7 .\end{array}$ & $\begin{array}{l}2 . \\
4 .\end{array}$ & $\begin{array}{l}3 . \\
4 .\end{array}$ \\
\hline \multicolumn{16}{|l|}{ Tiefe $\mathrm{m}$} \\
\hline 0 & 2 & 3 & 1 & 2 & 3 & 2 & 1 & 3 & 2 & 1 & 1 & 1 & 1 & 1 & 2 \\
\hline 5 & 2 & 3 & 1 & 2 & 3 & 4 & 1 & 1 & 1 & 1 & 1 & 1 & 1 & 1 & 2 \\
\hline 10 & 2 & 4 & 1 & 2 & 5 & 2 & 2 & 2 & 2 & 1 & 1 & 1 & 2 & 2 & 2 \\
\hline 20 & 2 & 3 & 1 & 3 & 3 & 3 & 3 & 2 & 1 & 1 & 1 & 1 & 2 & 1 & 2 \\
\hline 30 & 2 & 3 & 1 & 3 & 2 & 2 & 2 & 2 & 2 & 2 & 1 & 2 & 2 & 2 & 2 \\
\hline 50 & 2 & 3 & 1 & 2 & 2 & 4 & 1 & 1 & 4 & 2 & 1 & 2 & 2 & 2 & 2 \\
\hline 100 & 2 & 10 & 1 & 2 & 2 & 4 & 1 & 6 & 5 & 6 & 1 & 3 & 3 & 1 & 2 \\
\hline 150 & 3 & 4 & 1 & 3 & 2 & 4 & 2 & 4 & 6 & 3 & 5 & 5 & 8 & 2 & 2 \\
\hline 180 & 3 & 3 & 2 & 2 & 2 & 5 & 2 & 1 & 9 & 8 & 7 & 8 & 7 & 3 & 4 \\
\hline 200 & 4 & 3 & 2 & 4 & 2 & 4 & 3 & 1 & 18 & 15 & 14 & 10 & 12 & 4 & 4 \\
\hline
\end{tabular}

Tabelle 4.2. Mangan (Mn), in $\mu \mathrm{g} / \mathrm{l}$. Bodensee-Untersee, Stelle Berlingen.

Table 4.2. Manganese (Mn), expressed in $\mu \mathrm{g} / 1$. Lake Constance-Untersee, site Berlingen.

\begin{tabular}{|c|c|c|c|c|c|c|c|c|c|c|c|c|c|c|c|}
\hline & 1974 & & & & & & & & & & & & 1975 & & \\
\hline $\begin{array}{l}\text { Monat } \\
\text { Tag }\end{array}$ & & $\begin{array}{r}2 . \\
11 .\end{array}$ & $\begin{array}{r}3 . \\
11 .\end{array}$ & $\begin{array}{l}5 . \\
6 .\end{array}$ & $\begin{array}{r}5 . \\
29 .\end{array}$ & $\begin{array}{r}6 . \\
24 .\end{array}$ & 7. & $\begin{array}{r}8 . \\
12 .\end{array}$ & $\begin{array}{l}9 . \\
2 .\end{array}$ & $\begin{array}{l}10 . \\
30 .\end{array}$ & $\begin{array}{l}11 . \\
27 .\end{array}$ & 12. & $\begin{array}{r}1 . \\
20 .\end{array}$ & $\begin{array}{r}2 . \\
24 .\end{array}$ & $\begin{array}{r}3 . \\
23 .\end{array}$ \\
\hline \multicolumn{16}{|l|}{ Tiefe m } \\
\hline 0 & & 3 & 8 & 3 & 2 & 2 & & 3 & 2 & 1 & 9 & & 2 & 3 & 3 \\
\hline 5 & & 3 & 3 & 4 & 2 & 2 & & 4 & 2 & 2 & 11 & & 2 & 4 & 3 \\
\hline 10 & & 3 & 3 & 4 & 2 & 2 & & 4 & 3 & 1 & 11 & & 2 & 3 & 4 \\
\hline 15 & & 3 & 3 & 3 & 4 & 4 & & 6 & 2 & 2 & 6 & & 2 & 2 & 4 \\
\hline 20 & & 5 & 3 & 3 & 6 & 6 & & 5 & 2 & 1 & 3 & & 2 & 2 & 3 \\
\hline 30 & & 3 & 3 & 6 & 17 & 20 & & 30 & 42 & 1 & 17 & & 2 & 2 & 2 \\
\hline 40 & & 3 & 6 & 8 & 22 & 47 & & 36 & 45 & 1 & 5 & & 2 & 4 & 2 \\
\hline 44 & & 3 & 5 & 8 & 22. & 85 & & 42 & 50 & 12 & 7 & & 8 & 7 & 2 \\
\hline
\end{tabular}


Tabelle 4.3. Mangan (Mn) in $\mu \mathrm{g} / \mathrm{l}$. Greifensee, Stelle Maur-Greifensee.

Table 4.3. Manganese (Mn), expressed in $\mu \mathrm{g} / 1$. Greifensee, site Maur-Greifensee.

\begin{tabular}{lrrrrrrrrrrrrr}
\hline & 1974 & & & & & & & & & & & & \\
Monat & 1. & 2. & 3. & 4. & 4. & 5. & 6. & 7. & 8. & 9. & 10. & 11. & 12. \\
Tag & 7. & 4. & 4. & 2. & 29. & 27. & 24. & 22. & 19. & 16. & 15. & 11. & 9. \\
\hline Tiefe m & & & & & & & & & & & & & \\
0 & 27 & 59 & 56 & 20 & 10 & 5 & 4 & 6 & 0 & 1 & 8 & 14 & 41 \\
1 & 27 & 54 & 58 & 10 & 11 & 5 & 3 & 5 & 1 & 0 & 8 & 15 & 42 \\
2,5 & 25 & 55 & 58 & 13 & 10 & 5 & 5 & 6 & 1 & 2 & 8 & 16 & 41 \\
5 & 26 & 61 & 58 & 21 & 12 & 6 & 8 & 5 & 2 & 9 & 7 & 16 & 42 \\
10 & 30 & 63 & 58 & 32 & 19 & 20 & 36 & 45 & 78 & 63 & 10 & 16 & 45 \\
20 & 38 & 60 & 62 & 43 & 64 & 93 & 93 & 95 & 126 & 96 & 110 & 150 & 50 \\
30 & 210 & 118 & 132 & 500 & 350 & 280 & 105 & 160 & 156 & 145 & 153 & 225 & 100 \\
\hline
\end{tabular}

Tabelle 5.1. Kupfer (Cu), in $\mu \mathrm{g} / \mathrm{l}$. Bodensee-Obersee, Stelle Hagnau-Münsterlingen.

Table 5.1. Copper $(\mathrm{Cu})$, expressed in $\mu \mathrm{g} / \mathrm{l}$. Lake Constance-Obersee, site Hagnau-Münsterlingen.

\begin{tabular}{|c|c|c|c|c|c|c|c|c|c|c|c|c|c|c|c|c|}
\hline & 1974 & & & & & & & & & & & & 1975 & & & \\
\hline Monat & 1. & 2. & 3. & 4. & 5. & 6. & 7. & 8. & 9. & 10. & 11. & 12. & 1. & 2. & 3. & 4. \\
\hline Tag & 22. & 19. & 19. & 23. & 14. & 11. & 9. & 13. & 10. & 8. & 5. & 3. & 7. & 4. & 4. & 1. \\
\hline \multicolumn{17}{|l|}{ Tiefe $\mathrm{m}$} \\
\hline 0 & $<1$ & $<1$. & $<1$ & $<1$ & 1 & $<1$ & $<1$ & 1 & 1 & $<1$ & $<1$ & $<1$ & $<1$ & $<1$ & $<1$ & $<1$ \\
\hline 5 & $<1$ & $<1$ & $<1$ & $<1$ & $<1$ & $<1$ & $<1$ & $<1$ & $<$ & $<1$ & $<1$ & $<1$ & $<1$ & 1 & 1 & $<1$ \\
\hline 10 & $<1$ & $<1$ & 1 & $<1$ & $<1$ & $<1$ & $<1$ & $<1$ & 1 & $<1$ & $<1$ & $<1$ & $<1$ & $<1$ & $<1$ & $<1$ \\
\hline 20 & $<1$ & $<1$ & $<1$ & $<1$ & $<1$ & $<1$ & $<1$ & $<1$ & $<1$ & $<1$ & $<1$ & $<1$ & 1 & $<1$ & $<1$ & $<1$ \\
\hline 30 & $<1$ & $<1$ & $<1$ & $<1$ & $<1$ & $<1$ & 1 & 1 & $<1$ & $<1$ & $<1$ & $<1$ & 1 & $<1$ & 1 & $<1$ \\
\hline 50 & $<1$ & $<1$ & $<1$ & $<1$ & $<1$ & $<1$ & $<1$ & $<1$ & $<1$ & $<1$ & $<1$ & $<1$ & $<1$ & $<1$ & $<1$ & $<1$ \\
\hline 100 & $<1$ & $<1$ & $<1$ & $<1$ & $<1$ & $<1$ & $<1$ & $<1$ & 1 & $<1$ & $<1$ & 1 & $<1$ & 1 & $<1$ & $<1$ \\
\hline 150 & $<1$ & $<1$ & $<1$ & 7 & $<1$ & $<1$ & 1 & $<1$ & $<1$ & $<1$ & $<1$ & $<1$ & $<1$ & $<1$ & $<1$ & $<1$ \\
\hline 180 & $<1$ & 1 & $<1$ & $<1$ & $<1$ & $<1$ & $<1$ & $<1$ & $<1$ & $<1$ & $<1$ & $<1$ & 1 & 1 & 1 & $<1$ \\
\hline 200 & $<1$ & $<1$ & $<1$ & 1 & $<1$ & $<1$ & $<1$ & $<1$ & $<1$ & $<1$ & $<1$ & 1 & $<1$ & 1 & $<1$ & $<1$ \\
\hline
\end{tabular}

Tabelle 5.2. Kupfer $(\mathrm{Cu})$ in $\mu \mathrm{g} / \mathrm{l}$. Bodensee-Untersee, Stelle Berlingen.

Table 5.2. Copper $(\mathrm{Cu})$, expressed in $\mu \mathrm{g} / \mathrm{l}$. Lake Constance-Untersee, site Berlingen.

\begin{tabular}{|c|c|c|c|c|c|c|c|c|c|c|c|c|c|c|c|}
\hline $\begin{array}{l}\text { Monat } \\
\text { Tag }\end{array}$ & 1974 & $\begin{array}{r}2 . \\
11 .\end{array}$ & $\begin{array}{r}3 . \\
11 .\end{array}$ & $\begin{array}{l}5 . \\
6 .\end{array}$ & $\begin{array}{r}5 . \\
29 .\end{array}$ & $\begin{array}{r}6 . \\
24 .\end{array}$ & 7. & $\begin{array}{r}8 . \\
12 .\end{array}$ & $\begin{array}{l}9 . \\
2 .\end{array}$ & $\begin{array}{l}10 . \\
30 .\end{array}$ & $\begin{array}{l}11 . \\
27 .\end{array}$ & 12. & $\begin{array}{r}1975 \\
1 . \\
20 .\end{array}$ & 24. & $\begin{array}{r}3 . \\
23 .\end{array}$ \\
\hline \multicolumn{16}{|l|}{ Tiefe m } \\
\hline 0 & & 1 & 8 & 2 & 4 & 2 & & 5 & 1 & 3 & 6 & & 3 & 3 & 5 \\
\hline 5 & & 2 & 4 & 3 & 1 & 2 & & 6 & 1 & 3 & 8 & & 2 & 3 & 5 \\
\hline 10 & & 2 & 6 & 4 & 2 & 3 & & 7 & 1 & 3 & 8 & & 1 & 4 & 7 \\
\hline 15 & & 2 & 4 & 4 & 1 & 2 & & 10 & 1 & 4 & 9 & & 1 & 3 & 7 \\
\hline 20 & & 2 & 4 & 5 & 1 & 3 & & 7 & 1 & 4 & 10 & & 1 & 4 & 9 \\
\hline 30 & & 4 & 4 & 6 & 1 & 2 & & 7 & 1 & 5 & 12 & & $<1$ & 3 & 6 \\
\hline 40 & & 4 & 5 & 6 & 2 & 2 & & 10 & 1 & 5 & 7 & & 1 & 5 & 7 \\
\hline 44 & & 4 & 5 & 5 & 2 & 4 & & 9 & 1 & 2 & 8 & & 1 & 5 & 6 \\
\hline
\end{tabular}


Tabelle 5.3. Kupfer $(\mathrm{Cu})$ in $\mu \mathrm{g} / \mathrm{l}$. Greifensee, Stelle Maur-Greifensee.

Table 5.3. Copper (Cu), expressed in $\mu \mathrm{g} / 1$. Greifensee, site Maur-Greifensee.

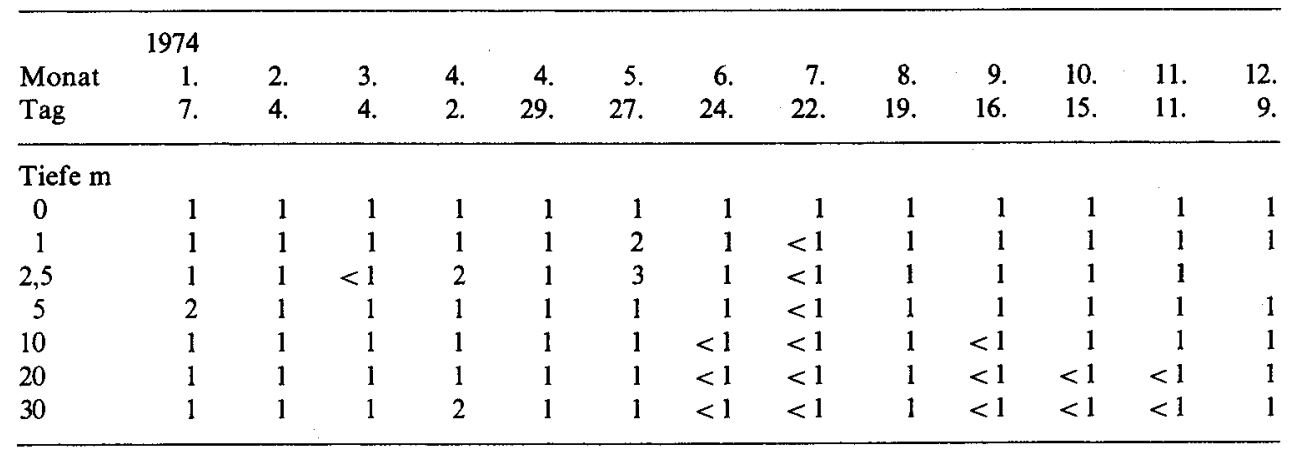

Tabelle 6.1. Zink ( $\mathrm{Zn})$, in $\mu \mathrm{g} / \mathrm{l}$. Bodensee-Obersee, Stelle Hagnau-Münsterlingen.

Tabelle 6.1. Zinc ( $\mathrm{Zn})$, expressed in $\mu \mathrm{g} /$ l. Lake Constance-Obersee, site Hagnau-Münsterlingen.

\begin{tabular}{|c|c|c|c|c|c|c|c|c|c|c|c|c|c|}
\hline \multirow{3}{*}{$\begin{array}{l}\text { Monat } \\
\text { Tag }\end{array}$} & \multicolumn{6}{|l|}{1974} & \multicolumn{7}{|c|}{1975} \\
\hline & 4. & 5. & 6. & 7. & 8. & 9. & 10. & 11. & 12. & 1. & 2. & 3. & 4. \\
\hline & 23. & 14. & 11. & 9. & 13. & 10. & 8. & 5. & 3. & 7. & 4. & 4. & 1. \\
\hline \multicolumn{14}{|l|}{ Tiefe m } \\
\hline 0 & 7 & 12 & 22 & 3 & 10 & 0 & 2 & 1 & 1 & 3 & 2 & 2 & 2 \\
\hline 5 & 3 & 3 & 2 & 11 & 1 & 0 & 1 & 5 & 2 & 2 & 3 & 4 & 1 \\
\hline 10 & 3 & 6 & 3 & 2 & 16 & 1 & 1 & 1 & 1 & 4 & 4 & 2 & 2 \\
\hline 20 & 5 & 5 & 6 & 4 & 3 & 1 & 1 & 1 & 3 & 3 & 2 & 2 & 1 \\
\hline 30 & 5 & 2 & 2 & 5 & 4 & 1 & 2 & 3 & 1 & 2 & 2 & 3 & 1 \\
\hline 50 & 14 & 1 & 9 & 3 & 2 & 1 & 2 & 1 & 1 & 2 & 3 & 2 & 2 \\
\hline 100 & 6 & 0 & 8 & 1 & 2 & 2 & 2 & 1 & 4 & 3 & 5 & 6 & 1 \\
\hline 150 & 14 & 2 & 9 & 7 & 1 & 1 & 2 & 1 & 1 & 1 & 3 & 3 & 1 \\
\hline 180 & 6 & 4 & 6 & 4 & 1 & 1 & 2 & 1 & 1 & 10 & 10 & 3 & 2 \\
\hline 200 & 20 & 5 & 6 & 11 & 1 & 8 & 2 & 1 & 2 & 9 & 3 & 2 & 3 \\
\hline
\end{tabular}

Tabelle 6.2. Zink ( $\mathrm{Zn}$ ) in $\mu \mathrm{g} / \mathrm{l}$. Bodensee-Untersee, Stelle Berlingen.

Table 6.2. Zinc $(\mathrm{Zn})$, expressed in $\mu \mathrm{g} /$ l. Lake Constance-Untersee, site Berlingen.

\begin{tabular}{|c|c|c|c|c|c|c|c|c|c|c|c|c|}
\hline & 1974 & & & & & & & & & 1975 & & \\
\hline $\begin{array}{l}\text { Monat } \\
\text { Tag }\end{array}$ & $\begin{array}{l}5 . \\
6 .\end{array}$ & $\begin{array}{r}5 . \\
29 .\end{array}$ & $\begin{array}{r}6 . \\
24 .\end{array}$ & 7. & $\begin{array}{r}8 . \\
12 .\end{array}$ & $\begin{array}{l}9 . \\
2 .\end{array}$ & $\begin{array}{l}10 . \\
30 .\end{array}$ & $\begin{array}{l}11 . \\
27 .\end{array}$ & 12. & $\begin{array}{r}1 . \\
20 .\end{array}$ & $\begin{array}{r}2 . \\
24 .\end{array}$ & $\begin{array}{r}3 . \\
23 .\end{array}$ \\
\hline \multicolumn{13}{|l|}{ Tiefe m } \\
\hline 0 & 5 & 9 & 6 & & 6 & 2 & 17 & 9 & & 4 & 5 & 4 \\
\hline 5 & 2 & 3 & 7 & & 8 & 2 & 10 & 17 & & 4 & 7 & 8 \\
\hline 10 & 4 & 3 & 12 & & 6 & 6 & 12 & 10 & & 3 & 6 & 9 \\
\hline 15 & 6 & 2 & 16 & & 7 & 1 & 16 & 15 & & 3 & 6 & 10 \\
\hline 20 & 5 & 6 & 10 & & 60 & 5 & 18 & 16 & & 4 & 6 & 5 \\
\hline 30 & 20 & 3 & 10 & & 10 & 3 & 21 & 20 & & 3 & 5 & 6 \\
\hline 40 & 12 & 3 & 15 & & 10 & 3 & 19 & 14 & & 3 & 8 & 4 \\
\hline 44 & 11 & 15 & 67 & & 9 & 4 & 18 & 19 & & 5 & 9 & 5 \\
\hline
\end{tabular}


Tabelle 6.3. Zink (Zn) in $\mu \mathrm{g} / \mathrm{l}$. Greifensee, Stelle Maur-Greifensee.

Table 6.3. Zinc (Zn), expressed in $\mu \mathrm{g} / \mathrm{l}$. Greifensee, site Maur-Greifensee.

\begin{tabular}{lrrrrrrrrrr}
\hline $\begin{array}{l}\text { Monat } \\
\text { Tag }\end{array}$ & 1974 & & & & & & & & & \\
& 2. & 4. & 5. & 6. & 7. & 8. & 9. & 10. & 11. & 12. \\
\hline Tiefe m & & & 27. & 24. & 22. & 19. & 16. & 15. & 11. & 9. \\
0 & 4 & 5 & 25 & 7 & 12 & 1 & 5 & 6 & 2 & 2 \\
1 & 6 & 9 & 2 & 3 & 4 & 1 & 1 & 7 & 2 & 2 \\
2,5 & 5 & 3 & 3 & 3 & 1 & 2 & 5 & 10 & 2 & 2 \\
5 & 17 & 4 & 3 & 14 & 3 & 2 & 2 & 4 & 3 & 2 \\
10 & 8 & 4 & 9 & 3 & 3 & 2 & 4 & 3 & 2 & 2 \\
20 & 23 & 20 & 4 & 3 & 2 & 4 & 3 & 3 & 2 & 2 \\
30 & 2 & 9 & 5 & 4 & 4 & 10 & 3 & 7 & 2 & 2 \\
\hline
\end{tabular}

Tabelle 7. Häufigkeit der Messwerte für $\mathrm{Cd}, \mathrm{Cr}, \mathrm{Pb}$.

Table 7. Measured values frequency for $\mathrm{Cd}, \mathrm{Cr}, \mathrm{Pb}$.

\begin{tabular}{|c|c|c|c|c|c|c|c|c|}
\hline \multirow{2}{*}{$\begin{array}{l}\text { Metall } \\
\text { Messwert } \\
\text { in } \mu \mathrm{g} / \mathrm{l}\end{array}$} & \multicolumn{4}{|c|}{ Anzahl Messungen } & \multicolumn{4}{|c|}{$\begin{array}{l}\text { Verteilung der Messwerte in } \% \\
\text { bezogen auf Total Messungen }=100 \%\end{array}$} \\
\hline & B & $\mathbf{U}$ & G & Total & B & $\mathrm{U}$ & $\mathrm{G}$ & Total \\
\hline $\mathrm{Cd}<0.1$ & 141 & 77 & 66 & 284 & 88 & 80 & 73 & 80 \\
\hline 0.1 & 19 & 16 & 24 & 59 & 12 & 17 & 26 & 19 \\
\hline 0.2 & 0 & 3 & 1 & 4 & 0 & 3 & 1 & 1 \\
\hline$\Sigma$ & 160 & 96 & 91 & 347 & 100 & 100 & 100 & 100 \\
\hline $\mathrm{Cr}<0.2$ & 37 & 21 & 13 & 71 & 23 & 22 & 32 & 26 \\
\hline 0.2 & 48 & 35 & 14 & 97 & 30 & 36 & 35 & 34 \\
\hline 0.3 & 30 & 14 & 0 & 44 & 19 & 15 & 0 & 11 \\
\hline 0.4 & 22 & 10 & 5 & 37 & 14 & 10 & 13 & 12 \\
\hline$\geqq 0.5$ & 23 & 16 & 8 & 47 & 14 & 17 & 20 & 17 \\
\hline$\Sigma$ & 160 & 96 & 40 & 296 & 100 & 100 & 100 & 100 \\
\hline $\mathrm{Pb}<1$ & 142 & 44 & 78 & 264 & 89 & 46 & 86 & 74 \\
\hline 1 & 18 & 43 & 10 & 71 & 11 & 45 & 11 & 22 \\
\hline 2 & 0 & 6 & 3 & 9 & 0 & 6 & 3 & 3 \\
\hline$\geqq 3$ & 0 & 3 & 0 & 3 & 0 & 3 & 0 & 1 \\
\hline$\Sigma$ & 160 & 96 & 91 & 347 & 100 & 100 & 100 & 100 \\
\hline
\end{tabular}

\section{SUMMARY}

Heavy Metals (Fe, $\mathrm{Mn}, \mathrm{Cd}, \mathrm{Cr}, \mathrm{Cu}, \mathrm{Pb}$ and $\mathrm{Zn}$ ) in the Pelagic Zone of the Lake of Constance (Obersee and Untersee) and the Greifensee

From the beginning of 1974 to spring 1975 the heavy metals $\mathrm{Fe}, \mathrm{Mn}, \mathrm{Cd}, \mathrm{Cr}, \mathrm{Cu}, \mathrm{Pb}, \mathrm{Zn}$ in the vertical profiles of the lake of Constance (Obersee and Untersee) and the Greifensee were determined each month by means of atomic absorption spectrometry (usually flameless). 
In the Untersee of Lake Constance and the Greifensee (both eutrophic, with oxygen depletion) $\mathrm{Fe}$ and especially $\mathrm{Mn}$ exhibited the usual seasonal fluctuations related to the redox conditions in the hypolimnion. No rise in the $\mathrm{Fe}$ and $\mathrm{Mn}$ content could be ascertained in the oligo-mesotrophic Obersee of the Lake of Constance.

$\mathrm{Cd}, \mathrm{Cr}, \mathrm{Cu}, \mathrm{Pb}$ and $\mathrm{Zn}$ showed no concentration patterns that revealed a dependence on season, vertical stratification or any other factor. In many of the measurements the $\mathrm{Cd}, \mathrm{Cr}, \mathrm{Cu}$ and $\mathrm{Pb}$ content lay below the minimum concentration measurable $(\mathrm{Cd} 0.1, \mathrm{Cr} 0.2, \mathrm{Cu} 1, \mathrm{~Pb} 1, \mathrm{Zn}$ about $1 \mu \mathrm{g} / 1$ under the measuring conditions observed).

In the Untersee of the Lake of Constance the $\mathrm{Cu}$ and $\mathrm{Zn}$ content lay considerably higher than in the Obersee of Lake Constance and the Greifensee. The $\mathrm{Cd}, \mathrm{Cr}$ and $\mathrm{Pb}$ content of the three lakes, however, did not differ significantly.

\section{RESUME}

Détermination des métaux lourds ( $\mathrm{Fe}, \mathrm{Mn}, \mathrm{Cd}, \mathrm{Cr}, \mathrm{Cu}, \mathrm{Pb}$ et $\mathrm{Zn}$ ) dans le zone pélagial du lac de Constance (Obersee et Untersee) et du Greifensee

Du début de 1974 jusqu'au printemps 1975 les métaux lourds $\mathrm{Fe}, \mathrm{Mn}, \mathrm{Cd}, \mathrm{Cr}, \mathrm{Cu}, \mathrm{Pb}, \mathrm{Zn}$ ont été déterminés chaque mois dans les profils verticaux du lac de Constance (Obersee et Untersee) et du Greifensee au moyen de la spectrométrie d'absorption atomique (en général sans flamme).

Dans l'Untersee du lac de Constance et dans le Greifensee (des lacs eutrophes et désoxygénés) une dépendence cyclique entre $\mathrm{Fe}$ et surtout $\mathrm{Mn}$ et les conditions redox dans l'hypolimnion a pu être établie. Aucune augmentation dans la teneur en Fe et Mn n'a été observée dans l'Obersee du lac de Constance (oligo-mésotrophe).

Quant à la teneur en $\mathrm{Cd}, \mathrm{Cr}, \mathrm{Cu}, \mathrm{Pb}, \mathrm{Zn}$, aucune dépendence des facteurs de la saison, la stratification verticale ou autre n'a pu être décelée de la courbe de concentration. Pour la plupart des valeurs mesurées, la teneur en $\mathrm{Cd}, \mathrm{Cu}, \mathrm{Cr}$ et $\mathrm{Pb}$ se situait en-dessous des concentrations minimales mesurables (Cd $0.1, \mathrm{Cr} 0.2, \mathrm{Cu} 1, \mathrm{~Pb} 1, \mathrm{Zn}$ environ $1 \mu \mathrm{g} / 1$ sous les conditions de mesure observées).

Dans l'Untersee du lac de Constance la teneur en $\mathrm{Cu}$ et $\mathrm{Zn}$ était considérablement plus élevée que dans le Obersee et le Greifensee. Le $\mathrm{Cd}, \mathrm{Cr}$ et $\mathrm{Pb}$ dans ces trois lacs ne montraient pas de divergences sensibles.

\section{VERDANKUNG}

Der Verfasser dankt Prof. H. Ambühl für wertvolle Anregungen bei der Abfassung des Textes, Herrn dipl. Chem. E. Szabo für die Organisation und Ausführung der Probenbeschaffung und für manche Diskussionen, Herrn dipl. sc. nat. H. Bührer für die EDV-Korrelationsberechnungen, den beteiligten Laboratorien für die Probenahmen sowie Frau W. Kempa und Frau S. Ochsner für die Ausführung der Metallbestimmungen.

\section{LITERATURVERZEICHNIS}

[1] AмвüнL, H., Versuch der Quantifizierung der Beeinflussung des Ökosystems durch chemische Faktoren: Stehende Gewässer, Schweiz. Z. Hydrol. 37, 35-52 (1975).

[2] Bilinski, H., Schindler, P., Stumm, W., und Zobrist, J., Kupfer und Blei in natürlichen Gewässern, Vom Wass. 43, 107-116 (1974).

[3] Bloesch, J., Sedimentation und Phosphorhaushalt im Vierwaldstättersee (Horwer Bucht) und im Rotsee, Schweiz. Z. Hydrol. 36, 71-186 (1974).

[4] Bradford, G.R., BaIR, F.L., und Hunsaker, V., Trace and Major Element Content of 170 High Sierra Lakes in California, Limnol. Oceanogr. 13, 526-529 (1968).

[5] BÜRGI, H.R., Die Wirkung von NTA auf das Wachstum des Phytoplanktons unter besonderer Berücksichtigung des Eisens als Mikroelement, Schweiz. Z. Hydrol. 36, 1-70 (1974). 
[6] Chen, K.Y., Young, C.S., JAN, T.K., und Rohatgi, N., Trace Metals in Wastewater Effluents, J. Wat. Pollut. Control Fed, 46, 2663-2675 (1974).

[7] Delfino, J.J., und Lee, G.F., Chemistry of Manganese in Lake Mendota, Wisconsin, Envir. Sci. technol. 2, 1094-1100 (1968).

[8] FörstNer, U., und Müller, G., Schwermetalle in Sedimenten des Bodensees, Naturwissenschaften 61,270 (1974).

[9] Förstner, U., und Müller, G., Schwermetalle in Flüssen und Seen (Springer-Verlag, Berlin, Heidelberg, New York 1974).

[10] Glass, G.E., und Podolski, J.E., Interstitial Water Components and Exchange across the Water Sediment Interface of Western Lake Superior, Verh. int. Ver. Limnol. 19, 405-420 (1975).

[11] Gorham, E., und SwaINE, D.J., The Influence of Oxidising and Reducing Conditions upon the Distribution of Some Elements in Lake Sediments, Limnol. Oceanogr. 10, 268-279 (1965).

[12] Groth, P., Untersuchungen über einige Spurenelemente in Seen, Arch. Hydrobiol. 68, 305-375 (1970).

[13] JACKSON, R.G., und Nichol, I., Factors Affecting Trace Element Dispersion in Lake Sediments in the Yellowknife A rea N.W.T., Canada, Verh. int. Ver. Limnol. 19, 308-316 (1975).

[14] Kennedy, V.C., Zellweger, G., und Jones, B.F., Filter-Pore-Size Effects on the Analysis of Al, $\mathrm{Fe}, \mathrm{Mn}, \mathrm{Ti}$ in Water, Wat. Resour. Res. 10, 785-790 (1974).

[15] Kimbald, K.D., Seasonal Fluctuations of Ionic Copper in Knights Pond, Massachusetts, Limnol. Oceanogr. 18, 169-171 (1973).

[16] Lodemann, C.K.W., und BUKenBerger, U., Schwermetallspuren im Bereich des oberen Neckars, GWF 114, 478-487 (1973).

[17] Robertson, D.E., The Adsorption of Trace Elements in Sea Water on Various Container Surfaces, Analyt. chim. Acta 42, 533-536 (1968).

[18] Santschi, P., und Schindler, P. W., Chemische Prozesse im Bielersee, Vom Wass, 43, 43-51 (1974).

[19] Smrrh, A.E., A Study of the Variation with $p H$ of the Solubility and Stability of Some Metal Ions at Low Concentrations in Aqueous Solution, Analyst 98, 65-68 (1973).

[20] Stumm, W., und Morgan, J.J., Aquatic Chemistry, Kap. 10-3 und 10-5 (Wiley Interscience, New York 1970).

[21] Welz, B., und Wiedeking, E., Einsatz der flammenlosen Atom-Absorption zur Bestimmung von Spurenmetallen in Wasser und Abwasser, Z. Anal. Chem. 264, 110-118 (1973).

Adresse des Autors:

Dr. H.R. Hegi, Eidg. Anstalt für Wasserversorgung, Abwasserreinigung und Gewässerschutz,

Uberlandstrasse 133, CH-8600 Dübendorf, Schweiz. 